\title{
Preparation of Monodispersed Nanoparticles of Transparent Conductive Oxides ${ }^{\dagger}$
}

\author{
Atsushi Muramatsu ${ }^{1 *}$, Kiyoshi Kanie ${ }^{1}$, Takafumi Sasaki ${ }^{2}$ and Masafumi Nakaya ${ }^{1}$ \\ ${ }^{1}$ Institute of Multidisciplinary Research for Advanced Materials (IMRAM), Tohoku University, Japan \\ ${ }^{2}$ Mitsui Mining \& Smelting Co., Ltd., Japan
}

\begin{abstract}
Generally, indium-tin-oxides (ITO) thin film is prepared by the sputtering process with ITO target, but only $20 \%$ of ITO yielded from the target is deposited on the substrate. Namely, about $80 \%$ ITO is exhausted by the deposition elsewhere far from the substrate. The recycling process of indium is limited so that ca. $20 \%$ ITO of the starting material is lost without any recovery. Even if the recycling of ITO has been carried out in this process, we should prepare ITO target of 5 times more than apparent use of ITO on film. If we change it to printing process from the sputtering, the reduction in ITO use is expected as ca. $50 \%$, considering the increase in film thickness by printing. Our target technology also includes ITO nanoink for the project. As a result, monodispersed ITO nanoparticles (NPs) with a cubic shape were fabricated by using quaternary ammonium hydroxide-assisted metal hydroxide organogels. These NPs have perfect uniformity in size with beautiful shape, and perfect single crystalline structure including Sn. As we were attempted to make thin film with ITO nanoink, it was successfully fabricated below $200 \mathrm{~nm}$ in thickness and the resistivity was drastically decreased below $1.0 \times 10^{-3} \Omega \mathrm{cm}$ after heat treatments. GZO nanoink as substitute of ITO has also been developed.
\end{abstract}

Keywords: uniform nanoparticles, transparent conductive oxides, indium tin oxide, shape control.

\section{Introduction}

Indium is well known to use as indium-tin-oxides (ITO) materials for transparent conductive oxides (TCO), which is often used in devices such as a flat panel display, touch-panel, solar cell, etc. Japan has an excellent advantage to produce ITO-relating devices, since Toyoha Mine in Sapporo, Japan, had supplied Indium as a top of world. But it was closed on March 31, 2006 mainly due to the economical reason. Before that, METI (Ministry of Economy, Trade and Industry, Japan) and Tohoku Univ. had started to discuss the project on substitute materials development of indium from 2004.

ITO thin film for devices is prepared by the sputtering process with ITO target, but only $20 \%$ of ITO yielded from the target is deposited on the substrate. Namely, about $80 \%$ ITO is exhausted by the deposition elsewhere far from the substrate as shown in Fig. 1. The recycling process is limited to be recovered so that only ca $80 \%$ ITO of the starting materials can be recycled but $20 \%$ is lost without any recovery. Even if the recycling of ITO has been carried out in this process, we should prepare ITO target of 5 times more than apparent use of ITO on film. If we change it to printing process from the sputtering, the reduction in ITO use is expected as ca. $50 \%$, considering the increase in film thickness by printing. Principally, the printing process results in the approximately $100 \%$ yield of film production from ITO nanoink, as shown in Fig. 2. As a result, our target technology included the development of ITO nanoink for this project. ITO nanoink was the next important technology for Japan, since the concept of printed electronics by use of nanoink has recently accepted as a novel engineering to make a functional thin film and/or advanced electronic circuits. Moreover, ITO film prepared by ink-jet of its

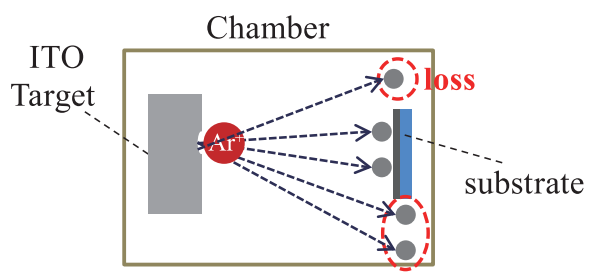

Fig. 1 ITO loss in sputtering process.

Received 16 May 2015; Accepted 10 August 2015

J-STAGE online 28 February 2016

Katahira 2-1-1, Aoba-ku, Sendai 980-8577, Japan

1-1-1 Hikoshimanishiyamacho, Shimonoseki-shi, Yamagachi, 750-0093 Japan

* Corresponding author: Atsushi Muramatsu;

E-mail: mura@tagen.tohoku.ac.jp

TEL: +81-22-217-5163 FAX: +81-22-217-5165 


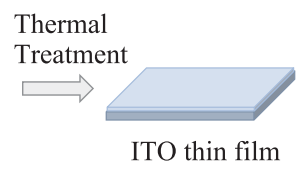

Fig. 2 Printing process of ITO nanoink.

nanoink has been expected as the transparent electrode of the next-generation solar cell. Namely, indium is well recognized as one of the most important resources for the future devices in Japan.

METI has started "Japan Rare Metal Substitute Materials Development Project" from the financial year of 2007, where Tohoku Univ. is the main university for research so that our project was also included as "Reduction of Indium use in transparent conducting electrodes." The sub-project, "Development of high performance ITO nanoparticles (NPs) for nano-ink," was composed of Tohoku University, ULVAC, Inc., Mitsui Mining \& Smelting Co., Ltd., DOWA Electronics Materials Co., Ltd., and Sharp Co., Ltd. This project has been carried out until FY2011. During it, several patents on ITO nanoink has been submitted. Besides, U.S.-Japan Roundtable on Rare Earth Elements Research and Development for Clean Energy Technologies, was held on November 18-19, 2010 on U.S. Department of Energy Lawrence Livermore National Laboratory so that we reported the investigation of ITO nanoink as a reduction technology of indium use.

The key technology of ITO nanoink is the production of ITO NPs precisely controlled in size, shape, and crystalline properties. However, ITO particles could not be prepared by hydrothermal synthesis because Sn-containing InOOH or $\mathrm{In}(\mathrm{OH})_{3}$ crystalline particles were once formed during aging even at higher temperature and at rather higher pressure but they could not be converted into ITO phase anymore. Considering the fact that alumina cannot be also prepared by the hydrothermal synthesis method, InOOH and $\operatorname{In}(\mathrm{OH})_{3}$ must be too stable to convert into ITO in aqueous media. Namely, we have reported the size and shape control of $\operatorname{In}(\mathrm{OH})_{3}$ nanoparticles by aminoacid assisted hydrothermal method. (Sasaki et al., 2009). Cubic-shaped $\operatorname{In}(\mathrm{OH})_{3}$ nanoparticles with the size of $50 \mathrm{~nm}$ were formed by addition of aspartic acid in the reaction solution. Hence, size- and shape-controlled $\operatorname{In}(\mathrm{OH})_{3}$ nanoparticles has been successfully prepared; however, the further thermal treatment is inescapable to obtain ITO nanoparticles.

We have found a novel solvothermal method to directly obtain ITO NPs with cubic morphology on 2008. (Endo et al., 2008). Remarkably, only by use of this procedure, highly crystalline cubic-shaped ITO NPs with narrow size distribution were successfully prepared, for the first time, through one-step process from a mixed ethylene glycol solution of indium and tin salts. (Sasaki et al., 2010)
In order to enhance the uniformity of as-prepared ITO, quaternary ammonium hydroxides were first applied for the solvothermal synthesis of monodispersed ITO NPs controlled in cubic morphology. Formation of a gel-like intermediate was a decisive factor for the precise control in size and shape. (Kanie et al., 2013) We also will show that their blue-colored compacts exhibits low resistivity as a transparent conductive oxide.

\section{Concept}

The unique properties of highly optical transparency of ITO in the visible region and controllable low resistivity have been utilized (Hartnagel, 1995) (Hamberg and Granqvist, 1986) so that ITO electrode is industrially prepared by sputtering or laser deposition method followed by chemical etching process. (Chiou et al., 1993) (Lin et al., 2008) (Gessert et al., 2009) On the other hand, since ITO is used in tandem with the penetration of a flat panel display, the world-wide demand for In as a TCO has been remarkably increased so that the saving and utilization of indium becomes task of pressing urgency. Furthermore, from the environmental point of view, such an energy-consumed system, including high-temperature and gas-phase deposition manufacturing process, fundamentally deserves a new look for the preparation of ITO electrode with low resistivity. In this regard, ITO nanoink, obtained by extremely stable dispersion of ITO NPs into medium, has been paid attention as an alternative to the conventional processes (Hong and Han, 2006) (Hong et al., 2008) (Bühler et al., 2007) However, TCO manufacture based on ITO nanoink contains many problems toward its practical use, as shown in Fig. 3. One of the most serious problems is the high resistivity of TCO electrodes from the nanoink, mainly because of the interfacial resistance between the particles, even if highly conductive ITO NPs are synthesized. As mentioned in Fig. 3, in order to reduce this resistance, the shape of the ITO particles can be designed as a cubic so that the face-to-face contact, 2-dimensional one, between particles is expected to decrease the resistance, compared with the point-topoint one between uncontrolled particles. In addition, for the high transparency and low haze, the particle size of ITO is to be below $100 \mathrm{~nm}$.

However, single-nanometer particles are not target materials in this study, as shown in Fig. 4. Even if monodispersed single-nanometer ITO particles are succeeded to produce, they are tremendously aggregated to form aggregates. Since the particle characteristics obey the apparent particle size, the size and shape of aggregates determines the quality and property. The control of size and shape of aggregates is theoretically impossible. Because of the higher surface reactibility, the corner become rounded 


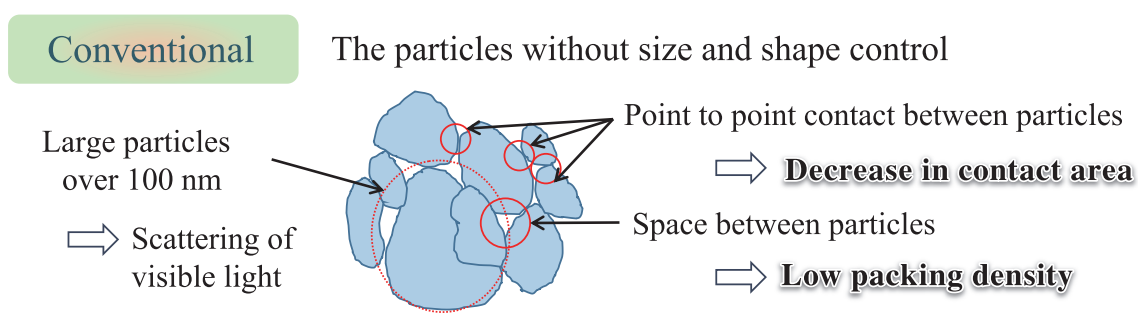

Increase in resistance

Our research

(1) Shape to promote conductive path $\Rightarrow$ Culbic-shape

(2) Size not to scatter visible light $\Rightarrow \leqslant 100 \underline{\mathrm{nm}}$

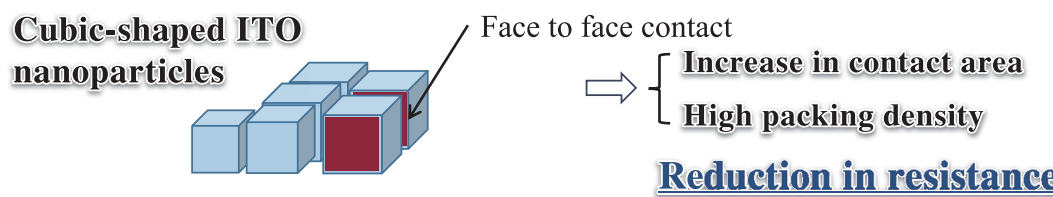

Fig. 3 Concept and design to synthesize ITO nanoparticles to be applied to the transparent conductive oxide electrodes.

Nano-ink does not require single-nanoparticles.

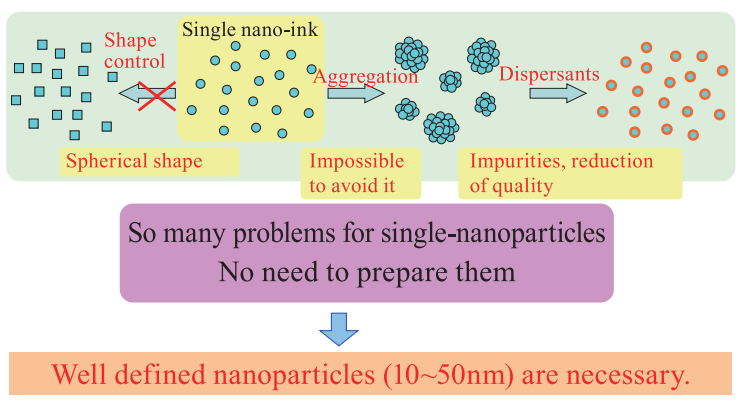

Fig. 4 Basic concept for uniform ITO particles synthesis.

off, even if cubic nanoparticles are initially formed. In addition, since the particle size is nearly equal to nuclei size, they are not well grown. The precise control in shape for single-nanoparticles are basically impossible. Since the dispersant is strongly and chemically adsorbed on the surface, the TCO properties get worse after calcination treatment. However, the dispersant is indispensable for well-dispersion of single-nanoparticles.

As a result, in order to achieve both the requirement of shape and size with higher cubic surface as well as less than $100 \mathrm{~nm}$ over $10 \mathrm{~nm}$, the preparation method of ITO NPs with high crystallinity, uniform composition, and well-defined particle morphology with monodispersed size distribution should be established.

\section{Gel-Sol method: Synthesis of uniform anatase-type $\mathrm{TiO}_{2}$ Nanoparticles}

The liquid-phase synthesis has much more advantages for the precise shape and size control of NPs, where the shape can be controlled by the addition of shape controller adsorbing selectively onto the specific surface and the size be by the strict separation of nucleation step, determining nuclei number, that is, the final particle size, from the particle growth, compared with other methods such as gas or solid-phase synthesis. (Sugimoto et al., 1998) (Kanie and Sugimoto, 2004) (Niederberger, 2007)

Owing to DLVO theory (Derjaguin and Landau, 1941) (Verwey and Overbeek, 1948), the dispersion and aggregation of fine particles have been estimated in rather diluted solution system. The concentrated solution leads to the tremendous aggregation between particles because of the compression of electrical double-layer, which contributes the dispersion of the particles through the electrostatic repulsion. In this regard, the dilute solution system is required for the monodispersed particle synthesis until our discovery of Gel-Sol method in the concentrated solution. The features of Gel-Sol method can be listed as follows. (Sugimoto et al., 2003) 1) Gel is a reservoir of metal ion so as to gradually supply it to the system under the rather low concentration of metal ions in solution phase. 2) Gel makes condensed viscous network in order to prevent the motion of particles to be aggregated each other by the anchoring particles in it. 3) Particles grow at the expense of the Gel until its complete consumption.

Here, as we illustrate a typical example of the Gel-Sol method in Fig. 5, the preparation method of monodispersed anatase-type $\mathrm{TiO}_{2}$ nanoparticles will be introduced as follows, since it is well-established procedure, based on the Gel-Sol transformation as above mentioned. This outstanding technology for the preparation of anatase nanoparticles precisely controlled in size and shape in- 


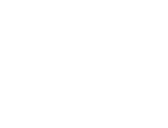

gatedes. 9 steps in the most important procedure. Namely, they are 1) the formation of rather stable Ti complex from Ti iso-propoxide with triethanol amine to inhibit the uncontrolled hydrolysis after the addition of water, 2) the fabrication of well stable gel network to avoid the tremendous aggregation between growing particles, 3) the strict control in the process temperature to strictly separate the nucleation stage from the particle growth one.

Established procedure will be shown in Fig. 5 in detail. The Ti stable complex composed of $\mathrm{Ti}$ and triethanol amine with $1 / 2$ in molar ratio. Since the formation rate of this complex is too slow to complete, it takes at least a whole day after Ti mixing with triethanol amine. If the complexation reaction is incomplete, the uncontrolled hydrolysis is promoted in the addition of water to the system. In that case, both the structure and composition must be inhomogeneous so that the monodispersed particles cannot be obtained.

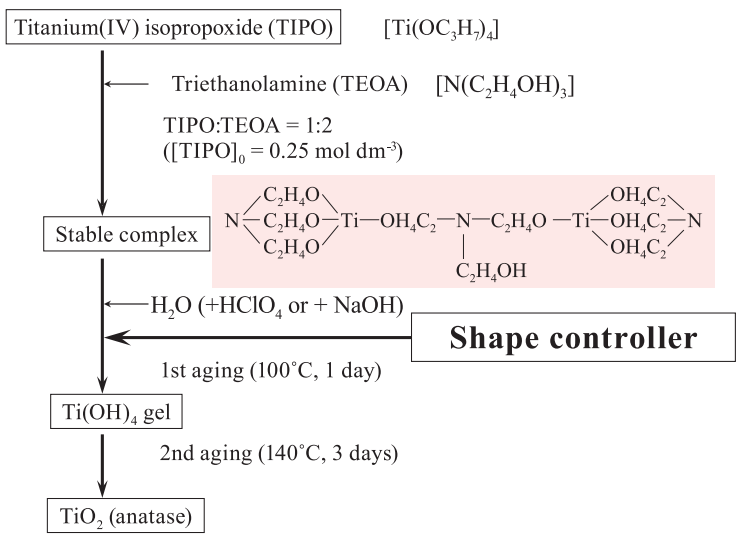

Fig. 5 Synthesis procedure of uniform $\mathrm{TiO}_{2}$ particles.

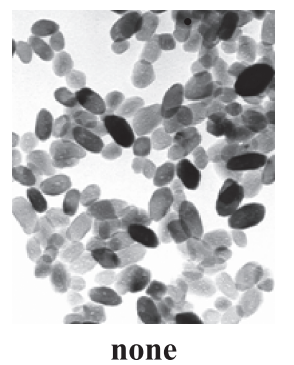

Init pH: 10.5

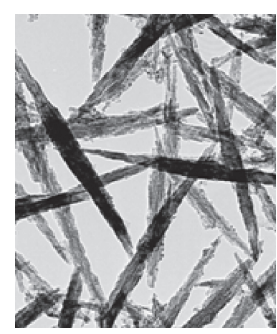

Ethylenediamine Init pH: 10.5

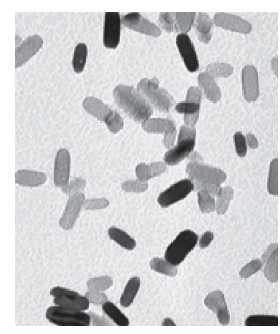

Glycine Init pH: 10.5

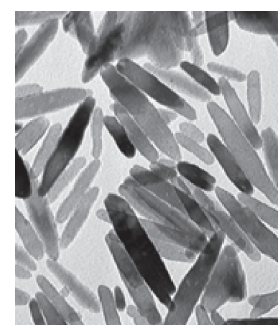

4-Aminobutylic Acid Init pH: 11.5

In order to apply them to a photocatalyst, the precise control of shape is the most important, because photocatalytic activities strongly depends on crystal facets (Kimijima et al., 2010), In Gel-Sol methods, the shape control can be also carried out simultaneously as the particle formation in their growth stage. Namely, the shape controller is added in the particle synthesis system. It is strongly adsorbed on the specific plane to disturb the crystal growth normal to the plane. As a result, the particles are formed, bounded by the plane adsorbed by the shape controller. Fig. 6 illustrates TEM photos of shape-controlled $\mathrm{TiO}_{2}$ nanoparticles. Basic reagents such as amines and amides were found effective to the shape control for anatase-type $\mathrm{TiO}_{2}$ nanoparticles, in comparison with oleic acid to shaping as cube. This synthesis procedure has been applied to the Ti-perovskite particles, such as $\mathrm{SrTiO}_{3}$ and $\mathrm{BaTiO}_{3}$, which showed characteristics unique in photocatalytic activities. (Kimijima et al., 2014)

The development of the synthesis method for monodispersed Ti-based oxide nanoparticles precisely controlled in size and shape directly led to the success in the production of uniform $\mathrm{Na}_{\mathrm{x}} \mathrm{K}_{\mathrm{y}} \mathrm{NbO}_{3}$ fine particles as a precursor of lead-free piezo materials for industrial use. (Kanie et al 2011) The niobate particle synthesis is basically the same as $\mathrm{TiO}_{2}$ preparation. In addition, it finally affects the development research for TCO nanoparticles, as described below.

\section{Application to direct ITO Synthesis}

Preparation methods of $\mathrm{In}_{2} \mathrm{O}_{3}$ or ITO NPs have been reported based on the combination of coprecipitation of

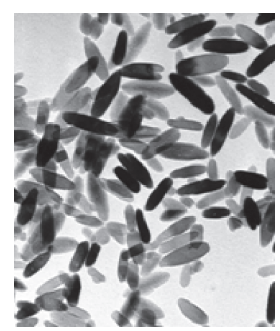

Succinic Acid

Init pH: 10.5

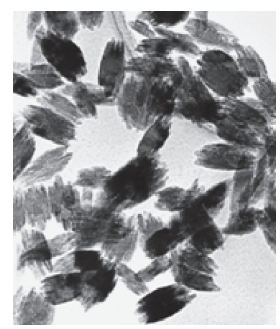

Oleic Acid

Init pH: 11.5

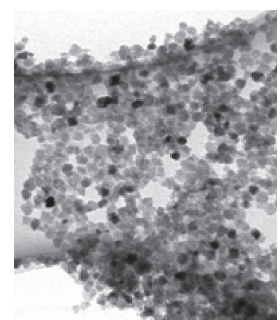

Gluconic Acid Init pH: 9.5

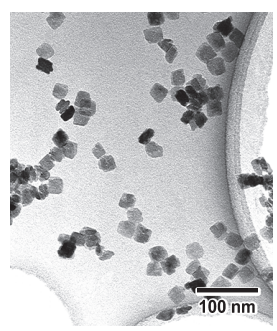

Oleic Acid Init pH: 9.9

Fig. 6 TEM photos of as-prepared uniform $\mathrm{TiO}_{2}$ particles. 


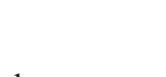

ndetal precursors and successive thermal treatment (Song et al, 2005) (Kim and Park, 2004), laser-induced fragmentation (Usui et al., 2006), solvothermal synthesis (Lee et al., 2005) (Yang et al., 2008a)), microwave-assisted synthesis (Okuya et al., 2007), emulsion techniques (Sujatha Devi et al., 2002), sol-gel synthesis (Aoki et al., 2006), and one-pot preparation of colloids (Seo et al., 2003) (Liu et al., 2005 (Lee et al., 2006) (Zhang et al., 2005) (Kanehara et al., 2009) (Gilstrap Jr. et al., 2008) (Gilstrap Jr. and Summers, 2009) However, the precise control of ITO NPs in size, shape, and structure simultaneously has never been reported, in particular, for monodispersed particle synthesis system. Namely, there is the technological difficulty for tin-doped indium oxide in the strict separation of nucleation and growth, and crystallization at rather low temperature below $300{ }^{\circ} \mathrm{C}$. Recently, we have reported size and shape control of $\operatorname{In}(\mathrm{OH})_{3}$ NPs by amino-acid assisted hydrothermal method(Sasaki et al., 2010). In this report, cubic-shaped $\operatorname{In}(\mathrm{OH})_{3}$ NPs with the size of $50 \mathrm{~nm}$ were formed by addition of aspartic acid in reaction solution. Since the thermal treatment is indispensable so as to obtain ITO particles at rather higher temperature, as-prepared ITO is aggregated and/or sintered so that the size and shape is changed. Even though monodispersed In$(\mathrm{OH})_{3}$ NPs are obtained, finally formed ITO NPs is not uniform. As a result, the monodispersed ITO NPs should be obtained by one-step synthesis.

ITO particles cannot be prepared directly by the hydrothermal synthesis, because $\operatorname{In}(\mathrm{OH})_{3}$ is not converted into ITO phase by solution-reprecipitation mechanism due to the extremely stable state of $\operatorname{In}(\mathrm{OH})_{3}$ phase. In this regard, the solvothermal system with use of an organic solvent, such as methanol, ethanol, and polyols, is applicable to the ITO synthesis by place of hydrothermal one, where water content can be reduced into the stoichiometric one for ITO formation. In the present paper, the direct synthesis of ITO NPs will be described by the combination of gel-sol and solvothermal method. Also, the present method will be applicable to the preparation of GZO, an alternative TCO for ITO.

\section{Experimental}

\subsection{First trial}

The first trial is unique in use of $\mathrm{NaOH}$ as a base as follows (Muramatsu, 2010). The reagent-grade indium (III) chloride tetrahydrate $\left(\mathrm{InCl}_{3} \cdot 4 \mathrm{H}_{2} \mathrm{O}\right)$, tin (IV) chloride pentahydrate $\left(\mathrm{SnCl}_{4} \cdot 5 \mathrm{H}_{2} \mathrm{O}\right)$, sodium hydroxide $(\mathrm{NaOH})$, ethylene glycol (EG), diethylene glycol (DEG), and 1-butanol $(\mathrm{BuOH})$ were used as received. The established procedure is as follows: $\mathrm{InCl}_{3}$ and $\mathrm{SnCl}_{4}$ were dissolved in $\mathrm{EG}$ with 0.50 and $0.050 \mathrm{~mol} \mathrm{~L}^{-1}$, respectively. In order to clarify the

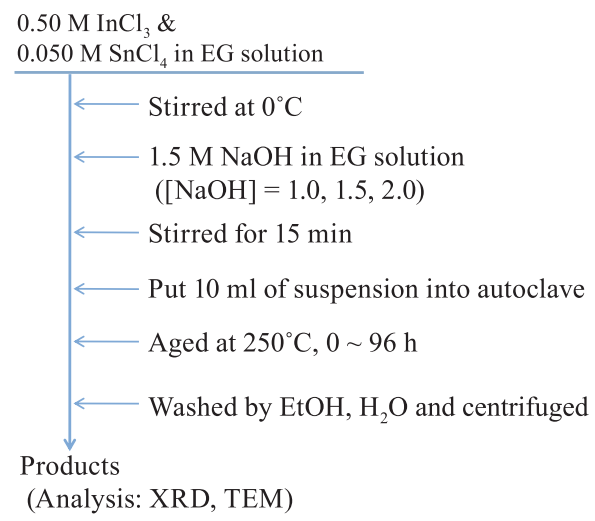

Fig. 7 Schematic drawing of the direct preparation of ITO nanoparticles by the solvothermal method with using ethylene glycol (EG) as a solvent.

effect of Sn concentration on the electric resistivity, $\mathrm{SnCl}_{4}$ concentration was varied in the range of $0-0.050 \mathrm{~mol} \mathrm{~L}^{-1}$ under the same $\mathrm{InCl}_{3}$ concentration at $0.50 \mathrm{~mol} \mathrm{~L}^{-1}$. An ethylene glycol solution of $\mathrm{NaOH}$ was also prepared and added to the same volume of $\mathrm{InCl}_{3}$ and $\mathrm{SnCl}_{4}$ ethylene glycol solution at $0{ }^{\circ} \mathrm{C}$ under agitation. The concentration of $\mathrm{NaOH}$ was adjusted to $1.0,1.5$, and $2.0 \mathrm{~mol} \mathrm{~L}^{-1}$. After $15 \mathrm{~min}$ stirring, the $10 \mathrm{~mL}$ suspension was transferred into a Teflon-lined autoclave (Parr Instrument, volume: $23 \mathrm{~mL}$ ) and aged at $250^{\circ} \mathrm{C}$ for 4 days. The standard conditions are molar ratio $\left[\mathrm{In}^{3+}\right] /\left[\mathrm{Sn}^{4+}\right]=10 / 1,\left[\mathrm{In}^{3+}\right] /$ $\left[\mathrm{OH}^{-}\right]=1 / 3$, and $250{ }^{\circ} \mathrm{C}$ for $12 \mathrm{~h}$. As-obtained product was purified by centrifugation $(18,000 \mathrm{rpm}, 10 \mathrm{~min})$, washed 3 times with ethanol, and dried at $60^{\circ} \mathrm{C}$ in an oven. Fig. 7 illustrates the standard procedure with use of $\mathrm{NaOH}$ as an alkaline reagent, we have developed first, to obtain ITO NPs by the present method.

\subsection{Established procedure}

As a result of all our attempts to prepare ITO, the established procedure is composed of the solvothermal synthesis and the Gel-Sol method as shown in Fig. 8 (Kanie et al., 2013). Namely, an EG solution of tetramethylammonium hydroxide (TMAH) in place of $\mathrm{NaOH}$ was prepared by using a $10 \mathrm{wt} \%$ TMAH methanol solution as follows. Initially, $100 \mathrm{~mL}$ of the TMAH solution was mixed with $25 \mathrm{~mL}$ of EG. Then, methanol was roughly removed by a rotary evaporator at $40{ }^{\circ} \mathrm{C}$ under $4 \mathrm{kPa}$, and the residue was evacuated at room temperature with stirring. Removal of methanol was confirmed by ${ }^{1} \mathrm{H}-\mathrm{NMR}$ measurement. Then, the TMAH concentration was determined by acid-base titration, and the concentration was adjusted from 1.0 to $2.5 \mathrm{M}$ by the dilution with EG. The synthesis procedure of ITO NPs with a cubic shape will be shown as follows. Initially, indium chloride tetrahydrate (In$\mathrm{Cl}_{3} \cdot 4 \mathrm{H}_{2} \mathrm{O}, 0.50 \mathrm{M}$ ) and tin chloride pentahydrate (Sn$\mathrm{Cl}_{4} \cdot 5 \mathrm{H}_{2} \mathrm{O}, 0.050 \mathrm{M}$ ) were dissolved in $\mathrm{EG}$ at room 
$0.50 \mathrm{M} \mathrm{InCl}_{3} \&$

Tetramethylammonium hydroxide (TMAH)

$\left(\mathrm{CH}_{3}\right)_{4} \mathrm{~N} \cdot \mathrm{OH}$

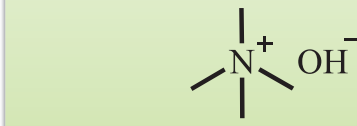

$\mathrm{OH}^{-}$ion resource
$0.050 \mathrm{M} \mathrm{SnCl}_{4}$ in Ethylene glycol (EG) solution

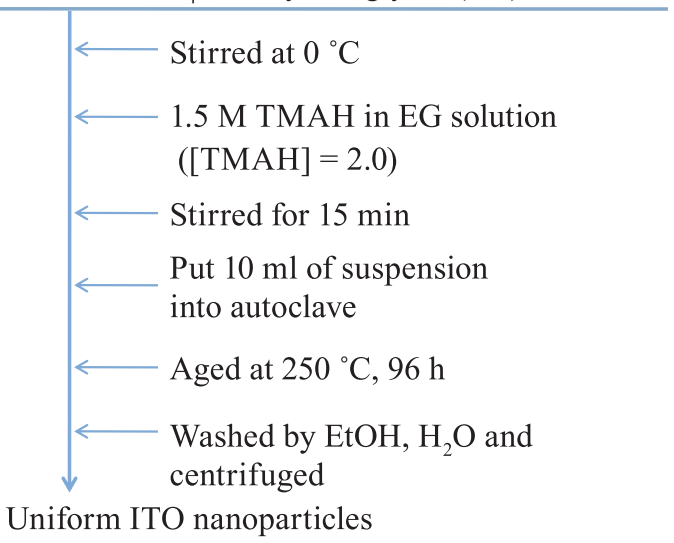

Fig. 8 Schematic drawing of the direct preparation of ITO nanoparticles by the combination of the solvothermal and Gel-Sol method with use of ethylene glycol (EG) as a solvent and TMAH as an alkaline reagent.

temperature. Then, $5.0 \mathrm{ml}$ of $2.5 \mathrm{M}$ TMAH solution in EG was poured into the same volume of as-prepared solution mixture of indium and tin under agitation at $0{ }^{\circ} \mathrm{C}$. The resultant solution was stirred at the temperature for 15 min, transferred into a Teflon-lined autoclave (Parr Instrument Company, Model 4749) and aged in an oven at $250{ }^{\circ} \mathrm{C}$ for $96 \mathrm{~h}$. As-obtained blue-coloured precipitates were collected by centrifugation (18,000 rpm, $10 \mathrm{~min})$ and washed three times with ethanol and one time with ion-exchanged water.

\subsection{Characterization}

The crystallographic structures of the dried powders were identified by a Rigaku Ultima-IV X-ray diffraction (XRD) measurement system using $\mathrm{Cu} \mathrm{K} \alpha$ radiation $(40 \mathrm{kV}$, $40 \mathrm{~mA}$ ) equipped with a D/teX Ultra detector. As-prepared particles were observed by a transmission electron microscope (TEM) using a Hitachi H7650 system with an acceleration voltage of $100 \mathrm{kV}$. High resolution TEM (HRTEM) analysis was carried out on a FEI TITAN 80-300. The particle surface was characterized by X-ray photoelectron spectroscopy using an ESCA-3400 (SHIMADZU Corporation).

\section{Results and Discussion}

\subsection{First trial with use of $\mathrm{NaOH}$}

In our first trial to prepare ITO directly by the solvothermal procedure, (Muramatsu et al., 2010) initially, the effect of $\mathrm{NaOH}$ concentration on the particle morphology and size in this system was investigated. The particle size was successfully controlled in the range from 10 to $50 \mathrm{~nm}$, as the initial $\mathrm{NaOH}$ concentration was decreased, (a)

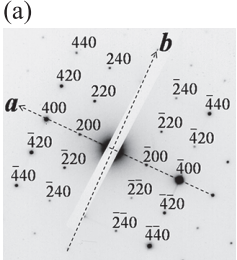

(b)

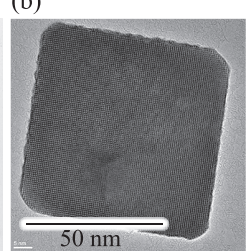

(c)

Fig. 9 Selected-area electron diffraction (a), respective TEM images (b), and HRTEM (c), of ITO nanoparticles shown in Fig. 10 (a).

possibly due to the increase in number of nucleus formed. Generally speaking, the rather higher alkaline condition is advantageous to the formation of the monomer, precursor complex, of metal oxide. ITO NPs were also prepared with different initial molar ratios of $\mathrm{Sn} / \mathrm{In}$ from 0 to 0.15 . In all cases, $\mathrm{In}_{2} \mathrm{O}_{3}$ and ITO NPs with cubic crystal structures were obtained. Trace amount of $\mathrm{SnO}_{2}$ was also formed only in the case with $\mathrm{Sn} / \mathrm{In}=0.15$, confirmed by XRD measurement. On the other hand, for $\mathrm{Sn} / \mathrm{In}=0.10$, $\mathrm{Sn} / \mathrm{In}$ molar ratio in as-prepared one ITO particle was found to keep in the same as the initial feed one, estimated by the energy dispersive X-ray (EDX) analysis.

Fig. 9 shows conventional TEM and high-resolution TEM (HRTEM) images with the electron diffraction (ED) spots. The ED spots as well as HRTEM analyses of the one particle shown in Fig. 9 (a) revealed that the crystal structure of the cubic shaped ITO NPs is basically single crystalline with the lattice parameter of the a-axis as $1.014 \mathrm{~nm}$, which is consistent with the a-axis of $\operatorname{In}_{2} \mathrm{O}_{3}$ with a cubic crystal structure (JCPDS No 06-0416) of $1.012 \mathrm{~nm}$. Thus, the incorporation of $\mathrm{Sn}$ ions into the crystal structure of $\operatorname{In}_{2} \mathrm{O}_{3}$ is not influenced to the change in the lattice parameter.

As a result of the effect of solvents, Figs. 10 and $\mathbf{1 1}$ show the TEM images and XRD patterns of the particles 


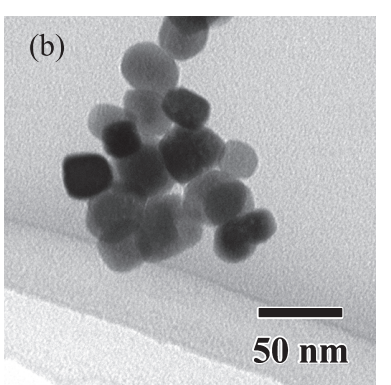

DEG

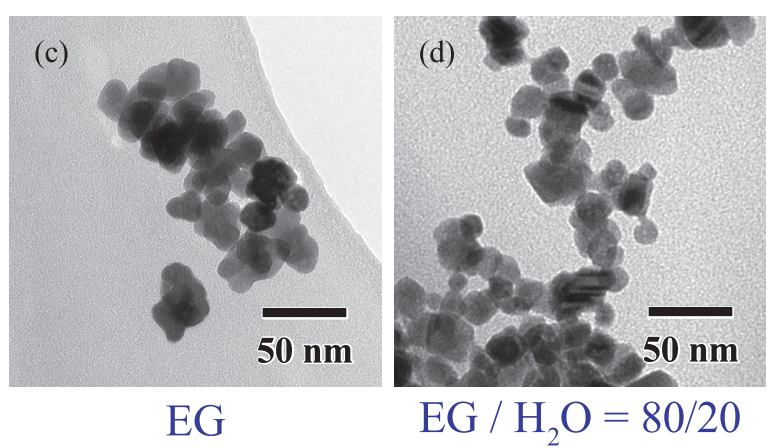

Fig. 10 TEM images of particles obtained in (a): $\mathrm{BuOH}$; (b): DEG; (c): EG; (d): $80 \mathrm{wt} \%$ EG aqueous solution.

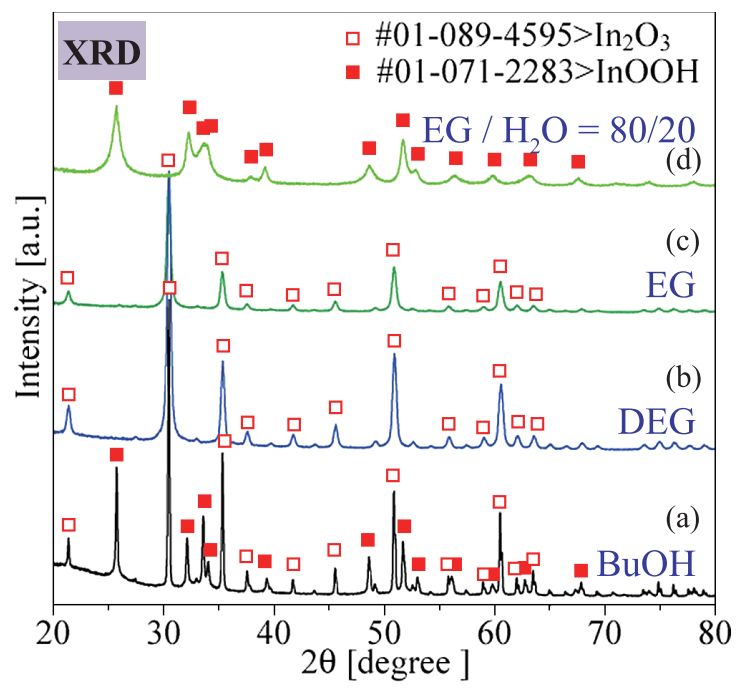

Fig. 11 XRD patterns of particles obtained in (a): $\mathrm{BuOH}$; (b): DEG; (c): EG; (d): $80 \mathrm{wt} \% \mathrm{EG}$ aqueous solution.

obtained in (a): EG; (b): diethylene glycol (DEG); (c): $80 \%$ EG aqueous solution; (d): butanol $(\mathrm{BuOH})$, respectively. Here, the molar ratios of $\mathrm{In}^{3+} / \mathrm{OH}^{-}$and $\mathrm{Sn} / \mathrm{In}$ were fixed as $1 / 3$ and 0.10 , respectively, with otherwise the standard condition, but aging time was $12 \mathrm{~h}$. As shown in Fig. 10 (a), irregular-shaped ITO NPs are obtained in EG aged for $12 \mathrm{~h}$, and the particle size is ca. $10 \mathrm{~nm}$. In the case of DEG, ITO NPs are also formed as a single phase, and the particle shape is basically cubic and the size is about $20 \mathrm{~nm}$. In contrast, the diffraction peaks of colorless powder obtained in an $80 \mathrm{wt} \%$ EG aqueous solution system (Fig. 11 (c)) are indexed to be an indium oxyhydrate
( $\mathrm{InOOH})$.

As a result of the comparison of EG-water and EG alone system, the addition of water seems inhibitory to the formation of ITO crystal. In the aqueous solution system of In salts, the formation of InOOH was strongly enhanced. One might consider that $\mathrm{InOOH}$ once formed during the reaction is hardly converted into $\mathrm{In}_{2} \mathrm{O}_{3}$ phase, because of the extremely low solubility of $\mathrm{InOOH}$. In other word, $\mathrm{InOOH}$ cannot play a role in an intermediate for $\mathrm{In}_{2} \mathrm{O}_{3}$ formation. In contrast, as has been reported elsewhere (Sugimoto et al., 1993) (Sugimoto et al., 2001), monodispersed hematite, $\alpha-\mathrm{Fe}_{2} \mathrm{O}_{3}$, particles in the concentrated Gel-Sol system, are formed through the 2-step phase transformation, namely, $\mathrm{Fe}(\mathrm{OH})_{3}$ to akaganeite, $\beta-\mathrm{FeOOH}$, and $\beta-\mathrm{FeOOH}$ to $\alpha-\mathrm{Fe}_{2} \mathrm{O}_{3}$, via a solution phase in the dissolution-reprecipitation process. In this case, $\beta$-FeOOH plays a role in the intermediate for $\alpha-\mathrm{Fe}_{2} \mathrm{O}_{3}$, because of the higher solubility.

The solid particles obtained by using $\mathrm{BuOH}$ as a solvent consist of $\mathrm{In}_{2} \mathrm{O}_{3}$ and $\mathrm{InOOH}$. It shows stepwise phase transition from metal hydroxides to give oxides by way of oxyhydroxides by the dissolution-precipitation is seen in the $\mathrm{BuOH}$ system. This behavior is commonly observed in aqueous systems to form metal oxides. In contrast, in the solvothermal system as shown here, direct formation of ITO solid particles is observed starting from amorphous-like indium hydroxides, $\mathrm{In}(\mathrm{OH})_{3}$ and $\mathrm{InOOH}$ phases are not detected as intermediates.

The temperature at aging in the autoclave was changed from 200 to $250{ }^{\circ} \mathrm{C}$ in ethylene glycol as a solvent, where aging period was fixed at $96 \mathrm{~h}$. Fig. 12 shows the effect of aging temperature on the ITO formation. Left-side of Fig. 12 illustrates TEM observation while right-side XRD. Judging from the TEM images, only an amorphous like product was formed at $200{ }^{\circ} \mathrm{C}$, but the shape-distinct solid particles were clearly observed over $220^{\circ} \mathrm{C}$. XRD patterns of the products also indicate not crystalline structure for $200{ }^{\circ} \mathrm{C}$ aging sample, in contrast to crystalline ITO phase over $220^{\circ} \mathrm{C}$. Thus, the crystallinity was obviously improved with increase in the aging temperature. Taking this remarkable temperature effect into consideration, the activation energy of the particle formation may be rather large so that the promoted formation rate can lead to the brisk nucleation and then the growth.

\subsection{Established synthesis method of ITO nanoparticles}

As has been reported (Kanie et al., 2013), Fig. 13 shows TEM images of the NPs obtained with different concentration of TMAH, (a) $0.50 \mathrm{M}$, (b) $0.75 \mathrm{M}$, (c) $1.0 \mathrm{M}$, and (d) 1.25 $\mathrm{M}$ under $0.25 \mathrm{M}\left[\mathrm{In}^{3+}\right] \quad 0.025 \mathrm{M}\left[\mathrm{Sn}^{4+}\right]$. XRD measurements revealed that all products could be assigned as an $\mathrm{In}_{2} \mathrm{O}_{3}$ with a cubic system of $\mathrm{Ia} 3$ (unit cell parameter: 

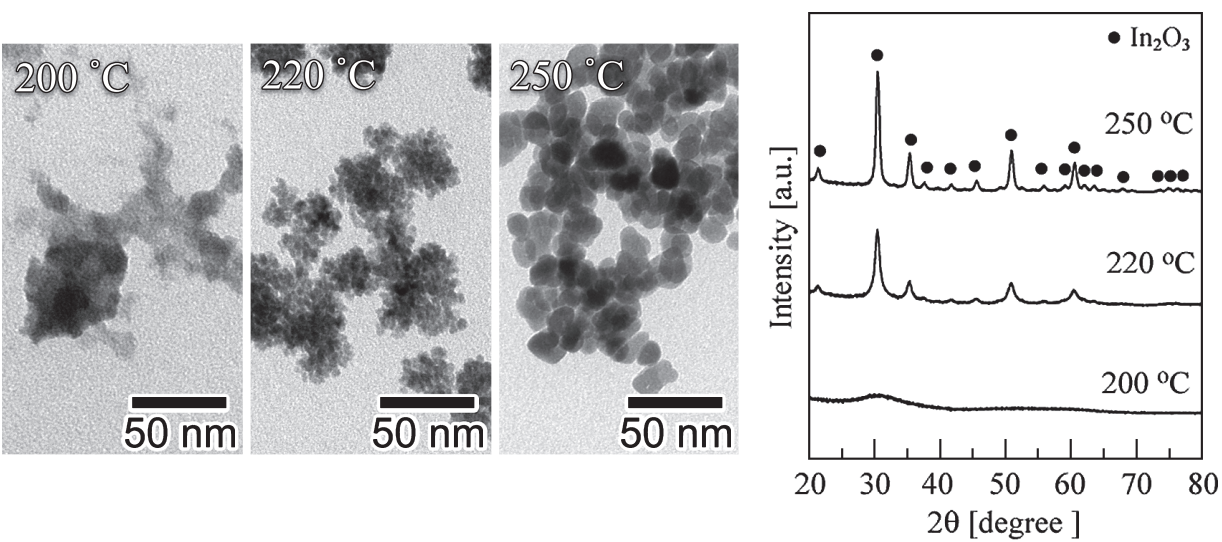

Fig. 12 Effect of the aging temperature of ITO particle synthesis under otherwise the standard condition. The temperature was varied in the range from 200 to $250^{\circ} \mathrm{C}$. (a) TEM observation, (b) XRD patterns.

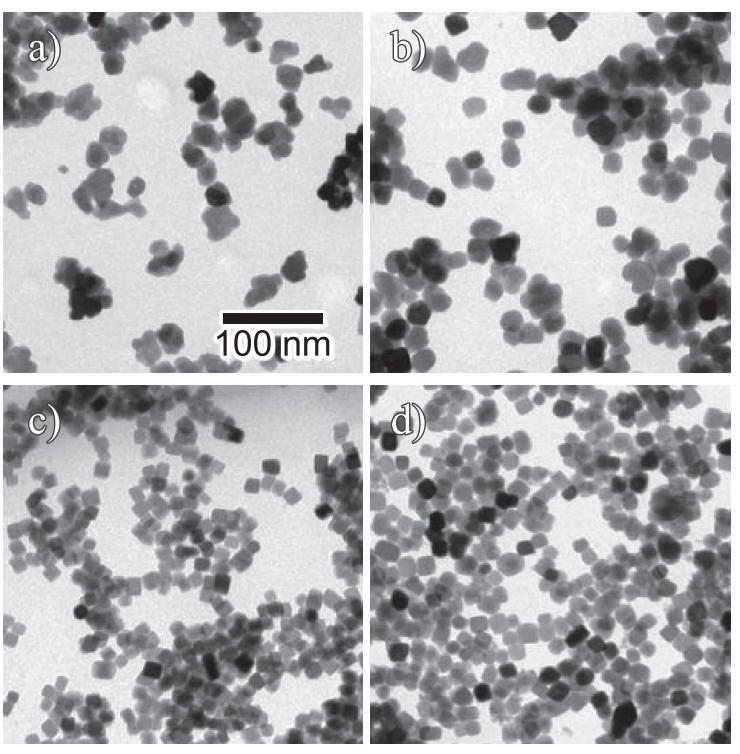

Fig. 13 TEM images of ITO NPs synthesized at $250{ }^{\circ} \mathrm{C}$ for $96 \mathrm{~h}$. The TMAH concentrations are (a) $0.50 \mathrm{M}$; (b) $0.75 \mathrm{M}$; (c) $1.0 \mathrm{M}$; (d) $1.25 \mathrm{M}$. The scale bar in (d) is common for all images.

$a=1.012 \mathrm{~nm}$ ) as a single-phase. As shown in Figs. 13 (a) and (b), inhomogeneous- and pseudocubic-shaped ITO NPs with round edges are formed at TMAH concentration of 0.50 and $0.75 \mathrm{M}$ in the solutions. In contrast, ITO NPs with a cubic shape with sharp edges are obtained for the TMAH concentration of more than $1.0 \mathrm{M}$ (Figs. 13 (c) and (d)). The average sizes of ITO NPs at different TMAH concentration are $26.1 \pm 6.5 \mathrm{~nm}(0.50 \mathrm{M}), 20.9 \pm 3.4 \mathrm{~nm}$ $(0.75 \mathrm{M}), 15.0 \pm 1.6 \mathrm{~nm}(1.0 \mathrm{M})$, and $17.3 \pm 1.9 \mathrm{~nm}$ $(1.25 \mathrm{M})$. Nucleation for metal oxide particle formation is generally promoted at higher alkaline condition in hydrothermal systems; since the formation of the metal hydroxide monomers precursory to oxide particles is accelerated. In the present solvothermal system, the initial mixing molar ratio of [TMAH] $/\left[\mathrm{In}^{3+}\right]$ was fixed to $2 / 1,3 / 1,4 / 1$, and $5 / 1$. In this regard, the decrease in the particle size by in-

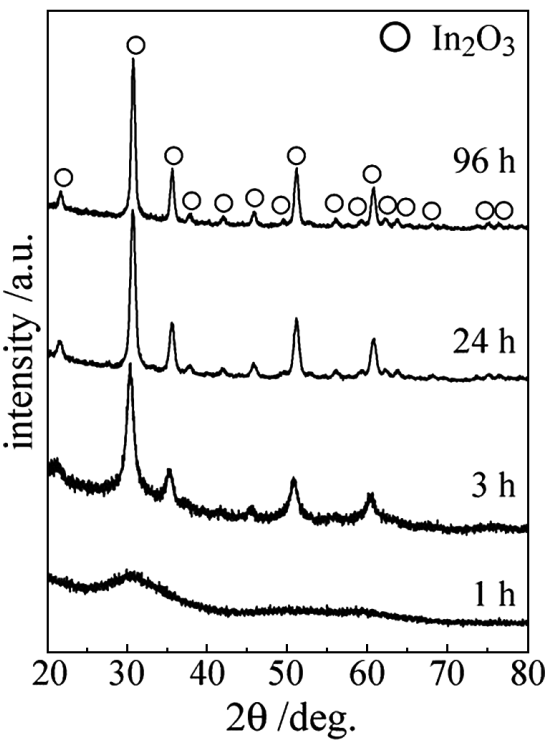

Fig. 14 XRD patterns of the precipitates formed by aging for (a) $1 \mathrm{~h}$; (b) $3 \mathrm{~h}$; (c) $24 \mathrm{~h}$; (d) $96 \mathrm{~h}$ at TMAH concentration of $1.0 \mathrm{M}$ in the solution.

crease in the TMAH concentration might be due to the promotion of metal hydroxides monomers in the initial reaction solution. The size distribution of particles obtained at 1.0 and $1.25 \mathrm{M}$ TMAH was found extremely narrow; actually, the standard deviation of the average size divided by the average size is calculated as $11.0 \%$. As a result, monodispersed ITO NPs were prepared with precise control in size and shape only by the present method.

Time evolution of the products at $250{ }^{\circ} \mathrm{C}$ under TMAH concentration of 1.0 M was investigated. As shown in Fig. 14, only broad halo-peaks in XRD analyses indicated the formation of an amorphous matter until $1 \mathrm{~h}$ aging. After $3 \mathrm{~h}$ aging, crystalline ITO phase was confirmed as a single phase, in contrast to no peak of crystalline $\operatorname{In}(\mathrm{OH})_{3}$ and InOOH phases. The diffraction peaks of ITO became sharpen with aging time so that crystallite size of the NPs 
nd oris

सो different aging time with 1.0 M TMAH are shown in Fig. 15, of which Fig. 15 (a) indicates an amorphous-like gel for the products obtained at $1 \mathrm{~h}$ aging. Judging from Fig. 15 (b), irregular-shaped ITO NPs are seemed to be formed in the amorphous-like gel matrix after $3 \mathrm{~h}$ aging. The particles grow at the expense of the amorphous gel, as shown in Fig. 15 (c). After $96 \mathrm{~h}$ aging, monodispersed ITO NPs with the cubic shape are finally formed as shown in Fig. 15 (d).

Fig. 16 illustrates a macroscopic change of the reactant mixture with aging time, including TEM images of the products obtained at each step. The reaction solution just
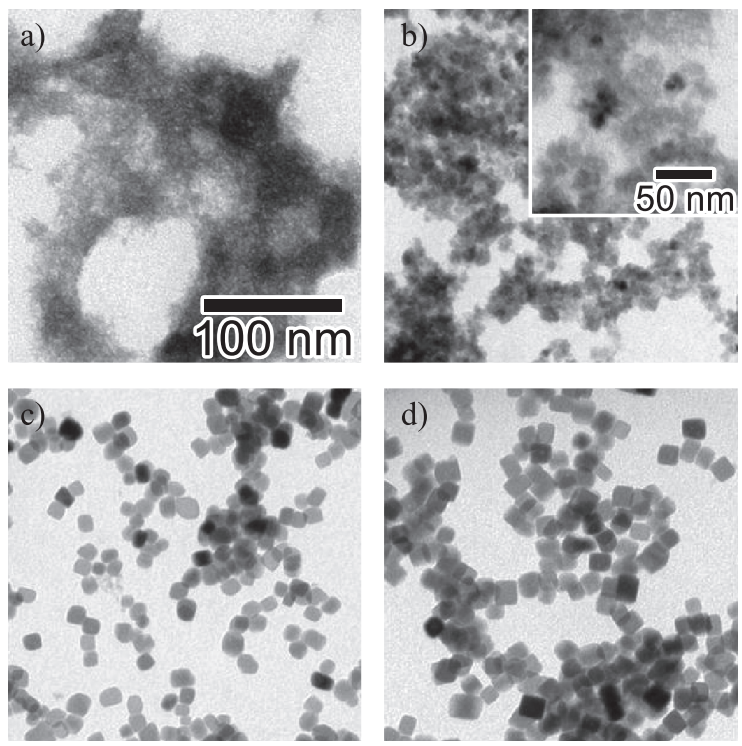

Fig. 15 TEM images of the precipitates formed by aging for (a) $1 \mathrm{~h}$; (b) $3 \mathrm{~h}$; (c) $24 \mathrm{~h}$; (d) $96 \mathrm{~h}$ at TMAH concentration of $1.0 \mathrm{M}$ in the solution. The scale bar in (a) is common for all images. after mixing both EG solutions of In/Sn and TMAH seemed homogeneous without any precipitates (Fig. 16 (a)). A viscous gel with pale yellow color was formed after $1 \mathrm{~h}$ aging at $250{ }^{\circ} \mathrm{C}$ (Fig. 16 (b)). As increasing aging time, the gel was gradually converted into a blue colored sol (Fig. 16 (c)), which suggests the formation of ITO NPs. The gel formed $1 \mathrm{~h}$ aging was further characterized by ICP, IR, and NMR analyses, after the gel was separated from solid and liquid parts by centrifugation. As a result of ICP measurement of the dried gel, the molar ratio of the $\mathrm{Sn} / \mathrm{In}$ was estimated as 0.038 , while indium ion concentration of the supernatant solution was $0.060 \mathrm{M}$. Namely, $75 \mathrm{~mol} \%$ of indium was used in the gel-intermediate transformed from the initial solution mixture. The actual concentration of the indium ions in the solution was kept at low state under aging. In this period, only particle growth became a predominant process to form monodispersed ITO NPs. FTIR analysis resulted in the distinct adsorption bands assigned to TMAH and EG. Since the amounts of TMAH and EG in the xerogels were quantitatively analyzed by ${ }^{1} \mathrm{H}-\mathrm{NMR}, 2.2 \mathrm{wt} \%$ of TMAH and $22.8 \mathrm{wt} \%$ of EG was included. As a result, the molar ratio of In:EG:TMAH was estimated as 1.0:0.8:0.05, respectively. The gel must be formed via the complex formation of indium hydroxides with EG and TMAH with hydrogen bonding network, confirmed by IR measurement of the xerogel. Yang et al also mentioned such chelation behavior of indium ions with polyols (Yang J. et al., 2008b). Thus, it is to be noted that TMAH may play a critical factor for the formation of the gel because such gel was not obtained by using $\mathrm{NaOH}$ as a basic agent (Sasaki et al., 2010). ITO NPs were formed, obeying the following gel-sol transformation mechanism, as a principle of the Gel-Sol method established in our laboratory. Initially, mixed solution of indium and tin salts dissolved in

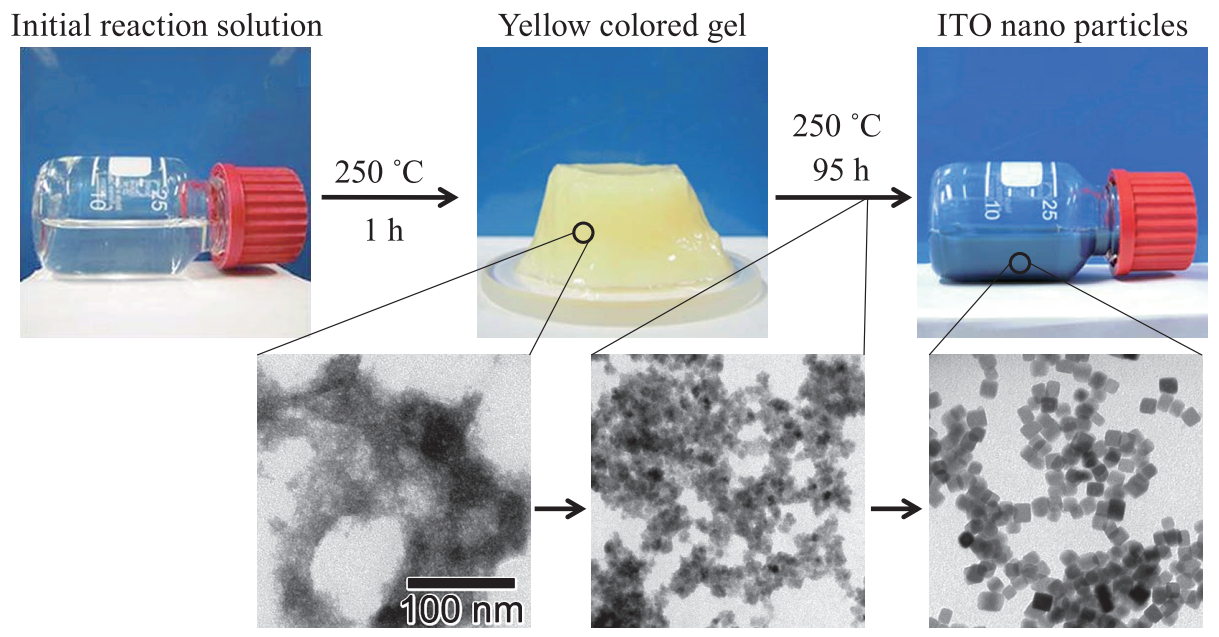

Fig. 16 A schematic illustration of time change of reaction solution at $250{ }^{\circ} \mathrm{C}$ in $1.0 \mathrm{M}$ TMAH. (a) A transparent precursor solution of the ITO NP synthesis. (b) A pale yellow viscous gel formed by aging for $1 \mathrm{~h}$ at $250{ }^{\circ} \mathrm{C}$. (c) A blue-colored ITO NP sol obtained by the present method. 
the Earsolution with TMAH was transformed into metal hydroxides at the early stage in aging time at $250{ }^{\circ} \mathrm{C}$. During aging, the viscous gel network was shaped via hydrogen bonding, as shown in Fig. 16 (b). Maybe, the nucleation of ITO were processed in the period of the gel matrixes formation. The growing particles were very tightly held on the gel matrixes so that NPs were grown through dissolution and recrystallization process at the expense of the gel. Such a gel-sol transformation of reaction solution was only observed at TMAH of 1.0 and 1.25 M, while the gel was not formed at lower TMAH concentration. Thus, the gel-sol transformation must be a key factor for synthesis of monodispersed ITO NPs.

The effect of $\mathrm{Sn} / \mathrm{In}$ ratio on the resultant particle size and shape was investigated so that cubic-shaped ITO NPS were also formed in the $\mathrm{Sn} / \mathrm{In}$ range from 0 to 0.20 . Predominant $\mathrm{In}_{2} \mathrm{O}_{3}$ and ITO NPs with the cubic crystal structures were obtained at $\mathrm{Sn} / \mathrm{In}$ from 0 to 0.15 without the formation of tin oxides such as $\mathrm{SnO}$ and $\mathrm{SnO}_{2}$. However, for $\mathrm{Sn} / \mathrm{In}=0.20$, a trace amount of $\mathrm{SnO}_{2}$ was identified. In this investigation, the average sizes with size distributions were $26.1 \pm 2.7 \mathrm{~nm}(\mathrm{Sn} / \mathrm{In}=0)$ and $23.9 \pm 2.8 \mathrm{~nm}$ $(\mathrm{Sn} / \mathrm{In}=0.020)$. The particle size was decreased with increase in $\mathrm{Sn} / \mathrm{In}$ ratio, possibly due to the acceleration of nucleation by tin. Such the size change with increase in Sn content was good agreement with the literature (Ba et al., 2006). The influence of species of the quaternary ammonium hydroxides on the ITO synthesis. Tetrabutylammonium (TBA) and tetrahexyl-ammonium (THA) hydroxides was also investigated. They also played the same role of promoting the formation of the cubic-shaped ITO NPs with sharp edges by way of gel-intermediates. The particle mean size and the distribution of the ITO NPs obtained by using $1.0 \mathrm{M}$ TBAH and THAH were $24.0 \pm 3.3$ and $25.5 \pm 4.6 \mathrm{~nm}$, respectively.

HRTEM and Fourier transform (FT) image of the cubic-shaped ITO NP (Fig. 15 (c)) are illustrated in Fig. 17. They clearly show the single-crystalline structure for the cubic-ITO NP. The lattice spacing parallel to exposing crystal plane calculated from Fig. 17 (b) is $0.50 \mathrm{~nm}$, corresponding to an interplanar distance of (200) planes of ITO with the cubic crystal system of Ia3. The FT pattern of Fig. 17 (b) means the particle growth perpendicular to $\{200\}$ plane. In general, particle morphology is controlled by the specific adsorption of additives, which regulates the growth rate of the particle in this direction. Thus, the reduction in the growth rate perpendicular to $\{200\}$ plane by specific adsorption of EG and TMAH leads to form the cubic-shaped ITO NPs in this system. The streaks, observed among each spots in the FT in the inset of Fig. 17 (b), suggested homogeneous substitutional doping of tin ions in the $\mathrm{In}_{2} \mathrm{O}_{3}$ crystal structure. Resistivity measurements of compacts prepared with ITO NPs with different $\mathrm{Sn} / \mathrm{In}$ molar ratio of $0,0.04,0.10$, and 0.15 were
$17.34,1.06,1.93$, and $2.67 \Omega \mathrm{cm}$, respectively. Thus, the optimized range in the tin doping amount was found about $0.04-0.10 \mathrm{~mol} \%$, and the minimal value of the powder resistivity was reached at $1.06 \Omega \mathrm{cm}$. It should be noted that the resistivity of the particles with the $\mathrm{Sn} / \mathrm{In}$ ratio of 0.10 was drastically decreased to $8.4 \times 10^{-2} \Omega \mathrm{cm}$ after the heat treatment at $300{ }^{\circ} \mathrm{C}$ for $30 \mathrm{~min}$ under air followed by $300{ }^{\circ} \mathrm{C}$ for 30 min under a $1 \% \mathrm{H}_{2}$ in $\mathrm{N}_{2}$ atmosphere. As a result, the cubic-shaped monodispersed ITO NPs with low resistivity synthesized in the present method, have large potential for the development of printable TCO nano-inks.

Finally, we were attempted to make thin film with ITO nanoink. As a result, ITO film below $200 \mathrm{~nm}$ in thickness was successfully fabricated and the resistivity of the particles $(\mathrm{Sn} / \mathrm{In}$ ratio of 0.10$)$ drastically decreased to below $1.0 \times 10^{-3} \Omega \mathrm{cm}$ by various heat treatments $1 \% \mathrm{H}_{2} / \mathrm{N}_{2}$ atmosphere. The value seems enough to apply to some specific region. Now, some companies are preparing commercial ITO nanoink after so many examinations for inkjet as shown in Fig. 18.

\subsection{GZO as a substitute ITO}

The substitute materials synthesis of ITO has been studied. Antimony-doped tin oxide (ATO), aluminum-doped zinc oxide (AZO), and gallium-doped zinc oxide (GZO) has been focused on in our laboratory. Gallium doped zinc oxide (GZO) has been expected as a substitute material of ITO for TCO. We also have been developed the synthesis method of GZO based on the similar one to ITO. For established procedure, $50 \mathrm{~mL}$ of $0.30 \mathrm{M} \mathrm{Zn}\left(\mathrm{NO}_{3}\right)_{2} \cdot 6 \mathrm{H}_{2} \mathrm{O}$ and $x \mathrm{~mol} \% \mathrm{Ga}\left(\mathrm{NO}_{3}\right)_{3} \cdot 5.8 \mathrm{H}_{2} \mathrm{O}$ solution $(x=\mathrm{Ga} / \mathrm{Zn}=0-30)$ in methanol was mixed well with the same volume of $0.60-1.8 \mathrm{M} \mathrm{NaOH}$. The mixed solution was aged at $200{ }^{\circ} \mathrm{C}$ for $1-48$ hours. For ATO, mixed solution of $\mathrm{SbCl} 5$ and $\mathrm{SnCl} 4$ After washing process with ethanol and distilled water, dried sample was evaluated with TEM.

Fig. 19 shows TEM photos GZO formed with 1,8 M $\mathrm{NaOH}$ after aging at $200{ }^{\circ} \mathrm{C}$ for 48 hours. GZO NPs were
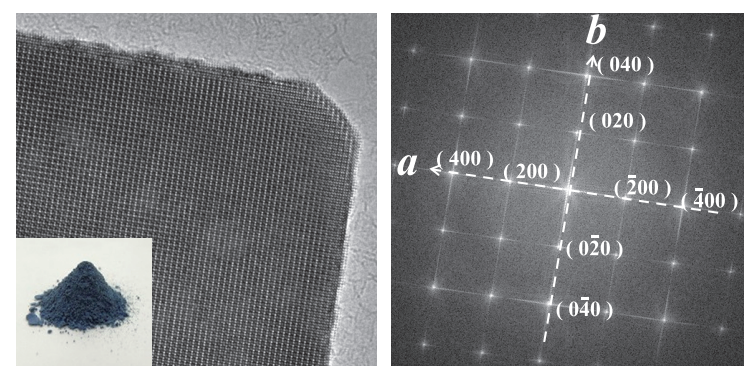

Fig. 17 (a) A HRTEM image of an ITO NP shown in Fig.15 (c); (b) A magnified and a FT images (inset) of the ITO NP exhibited in Fig. 15 (c). 


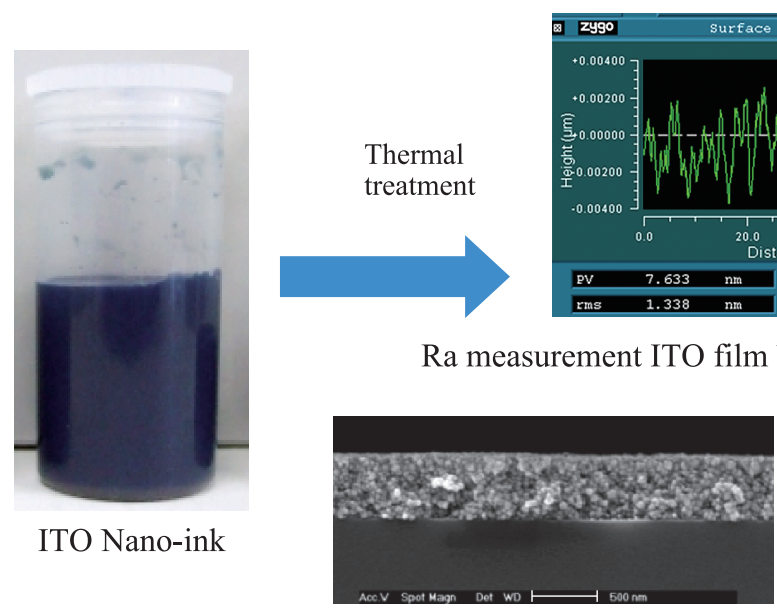

Cross section of ITO film

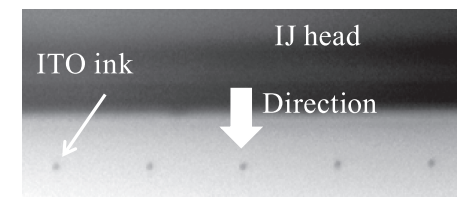

Ink-jet printing of ITO ink

Fig. 18 ITO nanoink for industrial use.
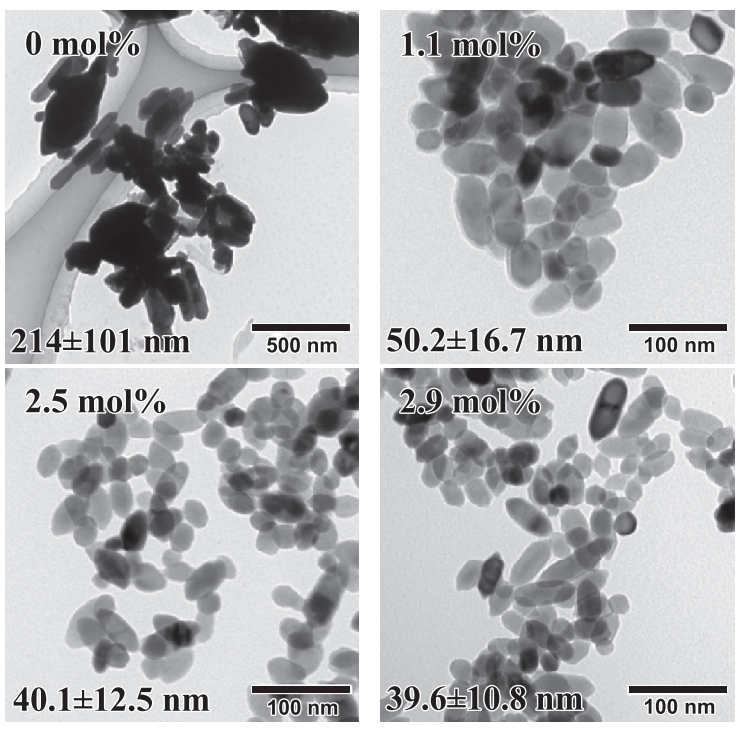

Fig. 19 GZO formed with $1.8 \mathrm{M} \mathrm{NaOH}$, aging time $24 \mathrm{~h}$.

successfully synthesized with narrow size distribution and precise control in Ga content. Judging from transmittance and resistivity of GZO thin film prepared by inkjet of GZO nanoink and then the thermal treatment with different temperature under air and then $\mathrm{H}_{2}$ for $1 \mathrm{~h}$ each, it is concluded that resistivity was monotonously decreased with increase in temperature, but the transmittance and haze was independent from it. As a result, our GZO nanoink can be applied for TCO as a substitute ITO.

\section{Conclusions}

Highly-crystalline monodispersed ITO NPs with a cubic shape were successfully synthesized by way of the combination of Gel-sol and solvothermal method by use of TMAH as a basic agent and EG as a solvent. The monodispersed ITO NPs were formed through dissolution and recrystallization process of the gel formed at early stage of aging. The initially formed gel consisted of indium hydroxide, EG, and TMAH played an important role for formation of monodispersed NPs. The HRTEM and FT analyses showed that the single crystalline cubic-shaped NP was formed by the reduction in the growth normal to $\{200\}$ plane. The FT analysis indicated Sn ions were substituted homogeneously in the ITO NP lattice. ITO nanoink was also prepared by the dispersion of ITO NPs into EG solution. Also, this combined method was applied to the synthesis of the other TCO NPs so that uniform GZO nanoparticles and their nanoink have been successfully prepared.

\section{Acknowledgements}

This work was financially supported by METI \& NEDO Rare Metal Substitute Materials Development Project and Industrial Technology Research Grant Program in 2011 (No. 11b15004d) from NEDO.

\section{References}

Aoki Y., Huang J., Kunitake T., Electro-conductive nanotubular sheet of indium tin oxide as fabricated from the cellulose template, Journal of Materials Chemistry, 16 (2006) 292297.

Ba J., Fattakhova Rohlfing D., Feldhoff A., Brezesinski T., Djerdj I., Wark M., Niederberger M., Nonaqueous synthesis of uniform indium tin oxide nanocrystals and their electrical conductivity in dependence of the tin oxide concentration, Chemistry of Materials, 18 (2006) 2848-2854.

Bühler G., Thölmann D., Feldmann C., One-pot synthesis of highly conductive indium tin oxide nanocrystals, Advanced Materials, 19 (2007) 2224-2227.

Chiou B.-S., Hsieh S.-T., R.F. Magnetron-sputtered indium tin 
an $\mathrm{or}^{\mathrm{s}}$

oxide film on a reactively ion-etched acrylic substrate, Thin WSolid Films, 229 (1993) 146-155.

Derjaguin B.V., Landau L., Theory of the stability of strongly charged lyophobic sols and of the adhesion of strongly charged particles in solutions of electrolytes, Acta Phys. Chim. URSS, 14 (1941) 633-662.

Endo Y., Sasaki T., Kanie K., Muramatsu A., Direct preparation and size control of highly crystalline cubic ITO nanoparticles in a concentrated solution system, Chemistry Letters, 37 (2008) 1278-1279.

Gessert T.A., Yoshida Y., Fesenmaier C.C., Coutts T.J., Sputtered $\mathrm{In}_{2} \mathrm{O}_{3}$ and ITO thin films containing zirconium, Journal of Applied Physics, 105 (2009) 083547.

Gilstrap Jr R.A., Summers C.J., Synthesis and analysis of an indium tin oxide nanoparticle dispersion, Thin Solid Films, 518 (2009) 1136-1139.

Gilstrap Jr R.A., Capozzi C.J., Carson C.G., Gerhardt R.A., Summers C.J., Synthesis of a nonagglomerated indium tin oxide nanoparticle dispersion, Advanced Materials, 20 (2008) 4163-4166.

Hamberg I., Granqvist C.G., Evaporated Sn-doped $\mathrm{In}_{2} \mathrm{O}_{3}$ films: Basic optical properties and applications to energyefficient windows, Journal of Applied Physics, 60 (1986) R123-R160.

Hartnagel H., Semiconducting transparent thin films, Institute of Physics Pub., Bristol [England]; Philadelphia, PA, 1995.

Hong S.-J., Han J.-I., Indium tin oxide (ITO) thin film fabricated by indium-tin-organic sol including ITO nanoparticle, Current Applied Physics, 6, Supplement 1 (2006) e206e210.

Hong S.-J., Kim Y.-H., Han J.-I., Development of ultrafine indium tin oxide (ITO) nanoparticle for ink-jet printing by low-temperature synthetic method, Nanotechnology, IEEE Transactions on, 7 (2008) 172-176.

Kanehara M., Koike H., Yoshinaga T., Teranishi T., Indium tin oxide nanoparticles with compositionally tunable surface plasmon resonance frequencies in the near-IR region, Journal of the American Chemical Society, 131 (2009) 1773617737.

Kanie K., Numamoto Y., Tsukamoto S., Takahashi H., Mizutani H., Terabe A., Nakaya M., Tani J., Muramatsu A., Hydrothermal synthesis of sodium and potassium niobates fine particles and their application to lead-free piezoelectric material, MATERIALS TRANSACTIONS, 52 (2011) 2119-2125.

Kanie K., Sasaki T., Nakaya M., Muramatsu A., Quaternary ammonium hydroxide-assisted solvothermal synthesis of monodispersed ITO nanoparticles with a cubic shape, Chemistry Letters, 42 (2013) 738-740.

Kanie K., Sugimoto T., Shape control of anatase $\mathrm{TiO}_{2}$ nanoparticles by amino acids in a gel-sol system, Chemical Communications, 14 (2004) 1584-1585.

Kim K.Y., Park S.B., Preparation and property control of nanosized indium tin oxide particle, Materials Chemistry and Physics, 86 (2004) 210-221.

Kimijima T., Kanie K., Nakaya M., Muramatsu A., Solvothermal synthesis of $\mathrm{SrTiO}_{3}$ nanoparticles precisely controlled in surface crystal planes and their photocatalytic activity,
Applied Catalysis B: Environmental, 144 (2014) 462-467.

Kimijima T., Sasaki T., Nakaya M., Kanie K., Muramatsu A., Photocatalytic activity of Ni-loaded $\mathrm{TiO}_{2}$ nanoparticles precisely controlled in size and shape, Chemistry Letters, 39 (2010) 1080-1081.

Lee C.H., Kim M., Kim T., Kim A., Paek J., Lee J.W., Choi S.-Y., Kim K., Park J.-B., Lee K., Ambient pressure syntheses of size-controlled corundum-type $\mathrm{In}_{2} \mathrm{O}_{3}$ nanocubes, Journal of the American Chemical Society, 128 (2006) 9326-9327.

Lee J.-S., Choi S.-C., Solvent effect on synthesis of indium tin oxide nano-powders by a solvothermal process, Journal of the European Ceramic Society, 25 (2005) 3307-3314.

Lin Y.C., Li J.Y., Yen W.T., Low temperature ITO thin film deposition on PES substrate using pulse magnetron sputtering, Applied Surface Science, 254 (2008) 3262-3268.

Liu Q., Lu W., Ma A., Tang J., Lin J., Fang J., Study of quasi-monodisperse $\mathrm{In}_{2} \mathrm{O}_{3}$ nanocrystals: Synthesis and optical determination, Journal of the American Chemical Society, 127 (2005) 5276-5277.

Muramatsu A., Sasaki T., Endo Y., Doi Y., Kanie K., Solvothermal Synthesis of ITO Nanoparticles Precisely Controlled in Size and Shape, Advances in Science and Technology, 62 (2010) 50-55.

Niederberger M., Nonaqueous sol-gel routes to metal oxide nanoparticles, Accounts of Chemical Research, 40 (2007) 793-800

Okuya M., Ito N., Shiozaki K., ITO thin films prepared by a microwave heating, Thin Solid Films, 515 (2007) 86568659.

Sasaki T., Endo Y., Nakaya M., Kanie K., Nagatomi A., Tanoue K., Nakamura R., Muramatsu A., One-step solvothermal synthesis of cubic-shaped ITO nanoparticles precisely controlled in size and shape and their electrical resistivity, Journal of Materials Chemistry, 20 (2010) 8153-8157.

Sasaki T., Nakaya M., Kanie K., Muramatsu A., Amino acid assisted hydrothermal synthesis of $\operatorname{In}(\mathrm{OH})_{3}$ nanoparticles controlled in size and shape, MATERIALS TRANSACTIONS, 50 (2009) 2808-2812.

Seo W.S., Jo H.H., Lee K., Park J.T., Preparation and optical properties of highly crystalline, colloidal, and size-controlled indium oxide nanoparticles, Advanced Materials, 15 (2003) 795-797.

Song J.E., Lee D.K., Kim H.W., Kim Y.I., Kang Y.S., Preparation and characterization of monodispersed indium-tin oxide nanoparticles, Colloids and Surfaces A: Physicochemical and Engineering Aspects, 257-258 (2005) 539542.

Sugimoto T., Chapter 8-control of particle characteristics, in: Sugimoto T. (Ed), Monodispersed particles, Elsevier, Amsterdam, 2001, pp. 376-388.

Sugimoto T., Khan M.M., Muramatsu A., Itoh H., Formation mechanism of monodisperse peanut-type $\alpha-\mathrm{Fe}_{2} \mathrm{O}_{3}$ particles from condensed ferric hydroxide gel, Colloids and Surfaces A: Physicochemical and Engineering Aspects, 79 (1993) 233-247.

Sugimoto T., Wang Y., Itoh H., Muramatsu A., Systematic control of size, shape and internal structure of monodisperse 
$\alpha_{-} \mathrm{Re}_{2} \mathrm{O}_{3}$ particles, Colloids and Surfaces A: PhysicochemiNCal and Engineering Aspects, 134 (1998) 265-279.

Sugimoto T., Zhou X., Muramatsu A., Synthesis of uniform anatase $\mathrm{TiO}_{2}$ nanoparticles by gel-sol method: 4. Shape control, Journal of Colloid and Interface Science, 259 (2003) 53-61.

Sujatha Devi P., Chatterjee M., Ganguli D., Indium tin oxide nano-particles through an emulsion technique, Materials Letters, 55 (2002) 205-210.

Usui H., Sasaki T., Koshizaki N., Optical transmittance of indium tin oxide nanoparticles prepared by laser-induced fragmentation in water, The Journal of Physical Chemistry B, 110 (2006) 12890-12895.

Verwey E.J.W., Overbeek J.T.G, Theory of the stability of lyo- phobic colloids. The interaction of particles having an electric double layer. Elsevier, New York-Amsterdam, 1948.

Yang J., Li C., Quan Z., Kong D., Zhang X., Yang P., Lin J., One-step aqueous solvothermal synthesis of $\mathrm{In}_{2} \mathrm{O}_{3}$ nanocrystals, Crystal Growth \& Design, 8 (2008a) 695-699.

Yang J., Li C., Quan Z., Zhang C., Yang P., Li Y., Yu C., Lin J., Self-assembled 3D flowerlike $\mathrm{Lu}_{2} \mathrm{O}_{3}$ and $\mathrm{Lu}_{2} \mathrm{O}_{3}: \mathrm{Ln}^{3+}(\mathrm{Ln}=$ Eu, Tb, Dy, Pr, Sm, Er, Ho, Tm) microarchitectures: Ethylene glycol-mediated hydrothermal synthesis and luminescent properties, The Journal of Physical Chemistry C, 112 (2008b) 12777-12785.

Zhang W., Huang Z., Li T., Tang Q., Ma D., Qian Y., A novel route to octahedral $\mathrm{In}_{2} \mathrm{O}_{3}$ particles exhibiting near band emission, Chemistry Letters, 34 (2005) 118-119.

\section{Author's short biography}

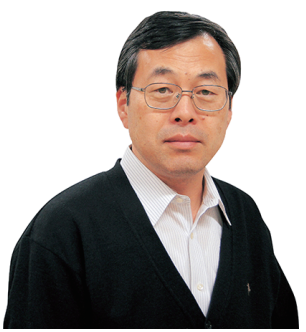

Dr.Atsushi Muramatsu is the Director and Professor of the Institute of Multidisciplinary Research for Advanced Materials, Tohoku University. The strategy of his laboratory, named as the Hybrid-Nanoparticle Lab., is to develop the novel synthesis method of highly functional nano-materials.

He graduated from the University of Tokyo in 1984 and then took the degree of Doctor of Engineering at the same university in 1988. He had a post of Research Associate at Tohoku University in 1988 and then Professor in 2001.

He was given the present position of the Director last spring.

\section{Kiyoshi Kanie}

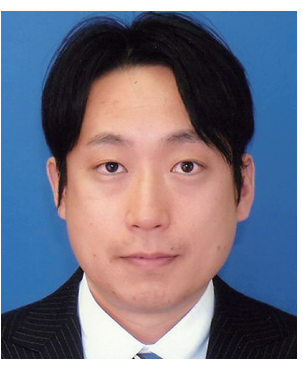

Dr. Kiyoshi Kanie, born in 1971, received his Bachelor's deg. from Nagoya Inst. of Tech. in 1994 and Master's deg. from Tokyo Inst. of Tech., 1996.

Then, he was appointed to an Assistant Prof. at the Univ. of Tokyo in 1998.

His research was exploration of materials with novel organic functionality through self-organization. In 2000, he received his Ph.D. degree from Kyoto Univ. He moved to the present institution as an Assistant Prof.in 2002 and promoted to an Associate Prof. in 2008. His current research includes the development of liquid-phase synthetic methods of functional inorganic nanoparticles preciously controlled in size and shape. He also focus on design and synthesis of functional organic materials. 


\section{Author's short biography}

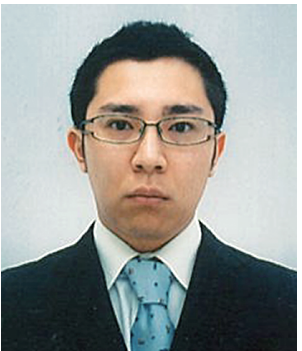

\section{Takafumi Sasaki}

Dr. Takafumi Sasaki, born in 1979 in Sendai, received his Ph.D. degree from Tohoku Univ. in 2008. Then, he worked in the Univ. as a post-doc researcher for 3 years. His main research subject was synthesis of functional inorganic nanoparticles and development in size control of the particles. In 2011, He moved his occupation to MITSUI MINING \& SMELTING CO., LTD.. He's engaged in work in Shimonoseki at present. His hobby is tour of hot springs in western Japan.

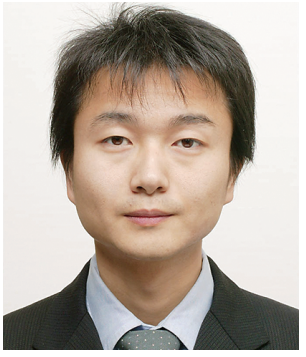

\section{Masafumi Nakaya}

Dr. Masafumi Nakaya, born in 1978, received his Bachelor's deg. from Kanazawa University, in 2002, Master's deg. from Japan Advanced Institute of Science and Technology, 2004, and Ph.D. degree from University of Tsukuba, 2007. Then, he worked in University of Tsukuba as a post-doc researcher for a year. He has worked in Tohoku University as an Assistant Professor since 2008. His main research theme is "Preparation of iron-based magnetic nanoparticles with tunable size and shape and control of their magnetic properties". 